\title{
ZUR SEMANTIK VON KOLLOKATIONEN ${ }^{1}$
}

\section{EINFÜHRUNG}

In der Sprachwissenschaft wird den Kollokationen seit mehreren Jahrzehnten großes Interesse entgegengebracht. Trotzdem konnte bisher keine eindeutige Bestimmung dieser spezifischen sprachlichen Erscheinung erreicht werden. Ein Grund dafür liegt in der Tatsache, dass Kollokationen für viele sprachwissenschaftliche Disziplinen relevant sind und jede ihre eigene Sichtweise beansprucht. So haben sich in der Sprachwissenschaft unterschiedliche Kollokationsauffassungen etabliert, die sich vom weiteren bis zum engeren Verständnis von Kollokationen erstrecken. ${ }^{2}$ Die weitere Auffassung fasst die Kollokation als Kookkurrenz im Sinne des Miteinandervorkommens von sprachlichen Elementen in Sätzen auf, wobei das Hauptkriterium die statistische Signifikanz ist. Im engeren Sinne stellt die Kollokation eine syntagmatische lexikalische Verbindung dar, die typisch, konventionell und rekurrent ist. ${ }^{3}$ Beiden Auffassungen ist gemeinsam, dass sie Kollokationen als bevorzugte Verbindung von Wörtern auf syntagmatischer Ebene betrachten, die häufig miteinander vorkommen. Da es sich um eine Kombination bestehender Begriffe handelt, zieht die engere Sichtweise zusätzlich Merkmale heran, um Kollokationen von anderen häufig miteinander vorkommenden Syntagmen $\mathrm{zu}$ unterscheiden. ${ }^{4}$ In Anlehnung an Reder (2006: 77) lassen sich diese Merkmale wie folgt bestimmen: konventionsbedingte Kookkurrenz der Kollokationsbestandteile, semantische Abhängigkeitsbeziehung in der Kollokation und eingeschränktes Beziehungspotenzial. Somit stützt sich die engere Kollokationsauffassung auf semantische Kriterien. Nachfolgend soll dies näher erläutert werden, wobei mittels einer funktionalen strukturell-syntaktischen Sicht auf die Kollokationsbestandteile die Kollokationen beschrieben werden sollen, um zum besseren Verständnis dieser komplexen sprachlichen Erscheinung beizutragen.

astojic@effri.hr

1 Diese Arbeit wurde von der Universität Rijeka im Rahmen des Projektes uniri-human-18-29 unterstützt.

This work has been fully supported by the University of Rijeka under the project number [unirihuman-18-29].

2 Einen ausführlichen Überblick über die geschichtliche Entwicklung der Kollokationsforschung bieten Bahns (1996), Bartsch (2004), Lehr (1996), Reder (2006), Konecny (2010), Stojić (2012) u. a.

3 Die engere Auffassung entspricht dem sog. phraseologischen Kollokationsverständnis und die weitere dem sog. frequenzorientierten Kollokationsbegriff, der für die Korpuslinguistik relevant ist.

4 Mehr dazu in Stojić/Košuta (2012). 


\section{KONVENTIONSBEDINGTE KOOKURRENZ}

Synchron betrachtet, bezeichnet die Kollokation einen Inhalt, der in einer bestimmten Sprachgemeinschaft innersprachlich begründet ist. So lautet beispielsweise die deutsche Verbindung Licht ausschalten im Englischen to switch the light off und im Kroatischen gasiti svjetlo ,Licht löschen'. Die gleiche außersprachliche Wirklichkeit wird in diesen Sprachen unterschiedlich versprachlicht, ohne dass sich dies semantisch erklären ließe, was den Schluss nahelegt, dass die Zuweisung willkürlich erfolgt (Stojić 2014: 82). Demnach bilden die Bestandteile der Kollokation zusammen eine Einheit, deren Bedeutung in einer bestimmten Sprachgemeinschaft konventionalisiert und sprachtypisch ist. Dies deutet auf Idiosynkrasie hin. Wenn die Wortverbindung idiosynkratisch ist, dann geht der Bildung dieser Wortverbindung ein unregelmäßiger Prozess voraus. Nach Konecny (2010: 167) ließe sich allein durch die Aufdeckung dieses Prozesses zeigen, ob ein bestimmtes Wort in der Verbindung einmal motiviert war oder nicht. Die Autorin veranschaulicht dies am Beispiel des Verbs lichten, das in der Bedeutung von hochziehen im Gegenwartsdeutschen nur in der Kollokation einen Anker lichten vorkommt. Es scheint also, dass diese Verbindung willkürlich ist. Bei diachroner Betrachtung zeigt sich jedoch, dass das Verb lichten etymologisch mit dem Syntagma leicht machen im Sinne von erleichtern verbunden ist. Dies kann mit dem Hochziehen des Ankers in Verbindung gebracht werden, da sich das Schiff mit dem hochgezogenen Anker leicht fortbewegen kann. Die Bedeutung scheint nach dieser Interpretation doch motiviert zu sein, sodass der Schluss naheliegt, dass diese Verbindung einmal frei war und mit der Zeit erstarrt ist. Die Kohäsion zwischen Kollokator und Basis bedingt, dass die Bedeutung des Verbs mit der Zeit verblasst und das Syntagma einen Anker lichten gegenwärtig als usuelle Wortverbindung zu bestimmen ist, die im Deutschen somit Idiosynkrasie aufweist. Nach Konecny (ebd. 176ff.) hängt der Grad an Idiosynkrasie von der Art der Kollokation ab, woraus folgt, dass es Kollokationen mit niedrigerem Grad an Idiosynkrasie und solche mit einem höheren Grad an Idiosynkrasie gibt. Der Grad selbst hängt von der Konzeptualisierung des Phänomens ab, die in der Kollokation versprachlicht wird, wobei der Grad an Idiosynkrasie durch eine diachrone Betrachtung der Bedeutung ermittelt werden kann. Daraus folgt, dass die Prozesse, die zu semantischen Änderungen führen, bestimmten Regeln folgen und daher nicht vollkommen idiosynkratisch sind. Die Frage, welche Prozesse in einer Sprache aktiviert werden und wie sie sprachlich ausgedrückt werden, ist jedoch idiosynkratischer Natur. Dies bedeutet, dass bei Kollokationen nur der Akt idiosynkratisch ist, durch den in einer Sprache etwas auf bestimmte Art und Weise versprachlicht wird.

\section{SEMANTISCHE ABHÄNGIGKEITSBEZIEHUNG}

Die Verbindbarkeit lexikalischer Einheiten beruht auf grammatischen und semantischen Prinzipien. Bei Kollokationen äußert sich die Grammatizität darin, dass die Bestandteile der Kollokation eine syntaktische Einheit bilden, demnach syntaktisch aufeinander bezogen sind. Nach der Art und Weise wie sich Kollokationen morphosyntaktisch verhalten, ist feststellbar, dass diese den allgemeinen grammatischen Regeln einer jeweiligen Sprache folgen. Das bedeutet, dass die einzelnen Bestandteile auf die 
gleiche Weise wie freie Wortverbindungen auch morphosyntaktisch verbunden werden. Die Bestandteile bilden syntaktische Muster. Hausmann (1985: 119) unterscheidet sechs Grundstrukturen im Deutschen, die auch auf die kroatische Sprache übertragbar sind (Stojić/Murica 2010: 116): 1. Verb + Substantiv (Basis), z. B. Gerechtigkeit suchen/tražiti pravdu, Sport treiben/baviti se sportom; 2. Adjektiv + Substantiv (Basis), z. B. schwarzer Pfeffer/crni papar, frisches Obst/svježe voće; 3. Substantiv (Basis) + Verb, z. B. das Telefon läutet/ telefon zvoni, der Hund bellt/pas laje; 4. Substantiv + Substantiv (Basis), z. B. eine Prise Salz/prstohvat soli, eine Tasse Kaffee/šalica kave; 5. Adverb + Adjektiv (Basis), z. B. vollkommen ruhig/potpuno miran, tödlich verletzt/ smrtno ranjen; 6. Adverb + Verb (Basis), z. B. richtig handeln/ispravno postupiti, scharf kritisieren/oštro kritizirati.

Die Beispiele zeigen, dass die äußere Grundstruktur der Kollokationen in beiden Sprachen, hier der deutschen und kroatischen, binär ist. Diese zweigliedrige Grundstruktur kann durch das Hinzufügen weiterer Elemente, welche die Bedeutung der einzelnen Bestandteile der Kollokation determinieren, erweitert werden. So besteht beispielsweise die Kollokation reiche Ernte einfahren aus drei Bestandteilen, die jedoch weiter in zwei Basiskollokationen zerlegt werden kann: reiche Ernte und Ernte einfahren. ${ }^{5}$

Betrachtet man die innere Struktur der Kollokationen, so können spezifische lexikalische Beziehungen ausgemacht werden, die häufig erst im Sprachkontrast auffällig sind. Vergleichen wir nämlich die Kollokation Ernte einfahren mit dem kroatischen Äquivalent prikupiti žetvu, Ernte einsammeln', so zeigt sich, dass der Bestandteil Ernte bzw. žetva seine Grundbedeutung beibehält und im Sprachkontrast übersetzungsäquivalent ist. Dieser Bestandteil ist somit semantisch unabhängig und wird deshalb in der deutschsprachigen Kollokationsforschung auch Basis genannt. Die andere Komponente, die mit der Basis gemeinsam auftritt, ist ihr hierarchisch untergeordnet, weil sie im semantischen Sinne von der Basis abhängt. Denn einfahren bzw. prikupiti kann, isoliert betrachtet, Unterschiedliches bedeuten. ${ }^{6}$ In Verbindung mit Ernte bzw. žetva wird das

5 Ähnlich argumentiert auch Hausmann, der Kollokationen lange Zeit ausschließlich als binäre Struktur definierte und erst in seinen späteren Arbeiten die zweigliedrige Grundstruktur auf eine sog. Tripelstruktur ausweitet, die aus zwei binären Kollokationen besteht, die zu einer Tripelkollokation verbunden sind (2004: 317ff.). Die Kollokationen Kritik an jmdm. üben und massive Kritik lassen sich auf die Tripelstruktur massive Kritik an jmdm. üben ausweiten.

6 Nach Duden-Online hat das Verb einfahren folgende Bedeutungen: 1. in etwas [hinein]fahren; fahrend in etwas gelangen; 2. (als Ernte) in die Scheune bringen; 3. (umgangssprachlich) erzielen, erwirtschaften; 4. durch heftiges Auffahren beschädigen, zerstören; 5. sich an ein bestimmtes Fahrzeug gewöhnen; 6 . durch entsprechende Fahrweise allmählich zu voller Leistungsfähigkeit bringen; 7. an das Ziehen eines Wagens gewöhnen; 8. zur Gewohnheit werden, sich einspielen; 9. (den einziehbaren Teil eines Apparates o. Ä.) mithilfe einer Mechanik nach innen bringen; 10. (Jägersprache) (von Fuchs, Dachs, Kaninchen o. Ä.) in den Bau [hinein]kriechen (www. duden.de/rechtschreibung/einfahren [12.11.2018]). Das Verb prikupiti hat nach dem OnlineWörterbuch Hrvatski jezični portal folgende Bedeutungen: 1. sabrati na jedno mjesto ono što se kupi ,an einen Ort zusammentragen'; 2. u manjoj mjeri, donekle okupiti (se) na jedno mjesto ,selten sich an einem Ort versammeln'; 3. (se) pren. sabrati se, doći k sebi ,übertr. mit sich: sich sammeln, zu sich kommen“ (http://hjp.znanje.hr/index.php?show=search [12.11.2018]). 
Element jedoch monosemiert. Diese Konstituente wird in Anlehnung an Hausmann (1984: 401) Kollokator genannt. Ausgehend vom Status der Basis und des Kollokators ergibt sich ein asymmetrisches Verhältnis in der Kollokation, in der grundsätzlich eine semantische Abhängigkeit des Kollokators von der Basis ausgemacht werden kann. Um die Bedeutung eines Kollokators zu definieren, ist mindestens eine Basis oder mehr erforderlich. Daher hat der Kollokator eine semantische Rolle bei der Bestimmung der Bedeutung einer Basis und nicht umgekehrt. Hausmann (1993: 475) erklärt die semantische Abhängigkeitsbeziehung in der Kollokation mit Hilfe des Begriffs Semiotaxis. Dieser stellt im Allgemeinen eine semantische Dimension auf der syntagmatischen Ebene dar. Hausmann deutet den Begriff jedoch um und bezeichnet den Kollokator vs. die Basis der Kollokation als semiotaktisch abhängig, während die Basis semiotaktisch autonom ist (ebd.). Der Ausgangspunkt für eine solche Reflexion ist die Fragestellung, ob die Bedeutung des Wortes autonom ist oder ein Kontext benötigt wird, der ein Wort semantisch spezifiziert. Nach Hausmann wird die Bedeutung eines Kollokators durch das Vorhandensein einer Basiskonstituente bestimmt, mit der es kontextuell verbunden ist. Mit Kontext meint er dabei das lexikalische Umfeld der Wörter auf der Ebene der parole und nicht den Situationskontext. Die Basis stellt immer eine referenzielle Größe dar, welche dazu dient, etwas zu benennen bzw. sprachlich zum Gegenstand zu machen. Sie überträgt ihre Grundbedeutung auf die Bedeutung der Kollokation, während die Bedeutung des Kollokators unterschiedliche Modifikationen erfahren kann. Sie kann spezifiziert, erweitert oder verengt, im schwachen Grade sogar auch umgedeutet werden (Stojić/Štiglić 2012: 267). Beim letzteren Typ sind zwei Arten von semantischen Beziehungen zu unterscheiden: metonymisch bedingter Wandel (z. B. eine Tafel Schokolade) und metaphorisch bedingter Wandel (z. B. Applaus ernten). Die Bedeutungsveränderung des Kollokators führt zur Polysemie, welche erst durch das Auftreten einer bestimmten Basis im Text disambiguiert wird. Wenn ein mehrdeutiges Lexem in einer Kollokation verwendet wird, differenziert seine Bedeutung von der Bedeutung seiner synonymen Partner und ist nicht mehr mehrdeutig. Das führt zur Unterscheidung zwischen kollokationsinterner und kollokationsexterner Bedeutung des Kollokators. Die kollokationsinterne Bedeutung ist die Bedeutung des jeweiligen Kollokators in der Kollokation und kollokationsextern die Bedeutung des Kollokators außerhalb der Kollokation, z. B. ist die Bedeutung von besuchen in der Kollokation den Arzt besuchen nicht dieselbe wie in der freien Verbindung die Großeltern besuchen (ebd.).

\section{EINGESCHRÄNKTES BEZIEHUNGSPOTENZIAL}

Das Prinzip des eingeschränkten und selektiven lexikalischen Kombinierens ist ein universales Merkmal jeder Sprache, während sich die Regeln einer solchen Verbindung und ihre semantischen Implikationen von Sprache zu Sprache unterscheiden (Borić 2000: 202). In der Regel werden Lexeme nach ihrer Bedeutung kombiniert, die auch mit der Bedeutung des Partners kompatibel sein muss. Diese durch Kompatibilität bedingte Verbindbarkeit ist die Kollokabilität, die bei Kollokationen lexikalischen Restriktionen unterliegt. Dies äußert sich darin, dass eine geringe paradigmatische Substituierbarkeit vorliegt, die gewisse Substitutionsrestriktionen zur Folge hat. So kann man 
das Verb bekommen in der Verbindung mit ein Kind nicht mit dem Synonym erhalten ersetzen. Diese Verbindung weist eine sprachinterne Bedingtheit auf. Welche Partner und wie viele ist, semantisch gesehen, in den meisten Fällen nicht vorhersagbar. ${ }^{7}$ Nach Kohn (1992: 374) bestimmen die Selektionsbeschränkungen in Abhängigkeit von den semantischen Merkmalen, welche Lexeme innerhalb bestimmter syntaktischer Rahmen miteinander verbunden werden können und welche nicht. Eine Verbindung wie grüne $K u h$ wäre zwar kein Verstoß gegen das System der deutschen Sprache, aber aufgrund des Weltwissens, das uns sagt, dass diesen sprachlichen Ausdrücken keine Referenten in der Welt entsprechen, ist eine solche Verbindung eher auf kreative Texte in Kunst oder Werbung beschränkt. Einige Einschränkungen sind durch die Kollokationsnatur bedingt, z. B. ranzige Butter. Das Lexem ranzig weist ein eingeschränktes Kollokationspotenzial auf, wobei sich die Frage stellt, ob diese Tatsache von den Substantiven Butter, Margarine, Öl, mit denen es gemeinsam auftritt, abhängt, oder es sich lediglich um einen konventionellen Gebrauch dieser Lexeme handelt.

Kollokationsrestriktionen wirken sich auf das Kollokationspotenzial einzelner Lexeme bzw. das Umfeld, in dem ein Lexem vorkommen kann, aus. Das Kollokationspotenzial selbst ergibt sich aus dem Bedeutungsumfang. Lexeme mit allgemeiner Bedeutung haben ein größeres Kollokationspotenzial als Lexeme mit spezifischer, enger Bedeutung (Pritchard 1998: 278). So unterscheiden sich z. B. die Adjektive eingefleischt und scharf im Kollokationspotenzial. Während scharf mit einer relativ großen Anzahl von Substantiven kombinierbar ist, z. B. Messer, Klinge, Soße usw., kann eingefleischt nur mit wenigen Substantiven wie Junggeselle, Optimist, Gegner verbunden werden. Das Kollokationsfeld ${ }^{8}$ von scharf ist somit größer als das von eingefleischt. Je niedriger das Kollokationspotenzial ist, desto größer ist der Grad der Lexikalisierung und Kohäsion.

Die Substitutionsrestriktionen wirken sich auch auf die semantische Kohäsion aus. Ist eine Konstituente der Kollokation nicht durch ihr Synonym zu ersetzen, verstärkt sich die semantische Kohäsion. So kann beispielsweise in der Wortverbindung starker Kaffee das Adjektiv stark nicht durch sein Synonym kräftig ersetzt werden. In anderen Kollokationen ist dies jedoch möglich, wie in starker Händedruck und kräftiger Händedruck, sodass es sich bei den letzten zwei Beispielen um eine semantische Verbindung schwacher semantischer Kohäsion handelt. Nach Teubert (1999) unterscheiden sich Kollokationen von freien Wortverbindungen gerade durch das Merkmal der semantischen Kohäsion. Je nach Grad der Kollokabilität kann die Intensität variieren. Je höher der Grad der Kollokabilität, desto geringer ist der Grad der Kohäsion (Iliescu 2006: 192).

7 Die Restriktionen beziehen sich nicht auf die morphosyntaktische Struktur. Dies bedeutet, dass zu einer Basis mehrere Kollokatoren derselben morphologischen Struktur gehören können. Es handelt sich also um funktionale Substituierbarkeit, die aber auf der semantischen Ebene Restriktionen aufweist.

8 Mit „Kollokationsfeld“ ist die Gesamtheit aller Synonyme mit gleichem Kollokationspotenzial gemeint. Mit den Kollokatoren erheben, zahlen, entrichten kollokieren Basen wie Steuern, Gebühren, Eintrittsgeld u. a. (Hausmann 1985: 127). 
Die paradigmatische Substituierbarkeit einer Komponente bestimmt auch die lexikalische Stabilität der Kollokation. Für eine Kollokation mit einem niedrigeren Grad an Stabilität kann eine Komponente, die Basis oder der Kollokator, substituiert werden, wie in Anforderung/Bedingung/Kriterium erfüllen. Der Grad der lexikalischen Stabilität hängt demnach vom Kollokationspotenzial des Kollokators ab.

Eine weitere Fragestellung betrifft auch die Wahl der einzelnen Bestandteile bzw. die Frage, welche Konstituente in einer Kollokation die andere wählt und wovon diese Wahl abhängt. Nach Konecny (2003: 105ff.) kann das Verhältnis zwischen den Kollokationskomponenten unilateral und bilateral sein. Ein einseitiges Verhältnis ist dann gegeben, wenn die Basis vom Kollokator gewählt wird, aber der Kollokator die Basis nicht impliziert. Im bilateralen Verhältnis impliziert der eine Bestandteil den anderen und umgekehrt. Der Kollokator ist in diesem Falle normalerweise ein expressives Lexem. Der Grad der Kollokabilität ist eingeschränkt oder unilateral, während der Grad der Kohäsion sehr hoch ist. Ein bilaterales Verhältnis zwischen den Kollokationskonstituenten ist beispielsweise in den Verbindungen ein Pferd wiehert, eine Kuh melken, ein Verbrechen begehen oder einen Streit schlichten gegeben. Verben wie wiehern haben einen unikalen Grad an Kollokabilität, während melken mit anderen Lexemen eine Kollokation eingehen kann. Aber das Verb melken geht ausschließlich mit solchen Lexemen eine Kollokation ein, die zum selben semantischen Feld gehören (ein Tier, das gemolken werden kann). Die Verben begehen und schlichten werden wiederum nur selten kollokationsextern verwendet. Das bilaterale Verhältnis zeigt sich auch in der Tatsache, dass die Basis manchmal weggelassen werden kann. So kann zum Beispiel das Verb schlichten ohne Basis verwendet werden. Das unilaterale Verhältnis erscheint in Kollokationen, deren Kollokator einen hohen Grad an Kollokabilität, eine geringe Expressivität und schwache Intension aufweist. Die Basis und der Kollokator haben kein gemeinsames Sem. Konecny (ebd.) veranschaulicht das unilaterale Verhältnis am Beispiel der italienischen Kollokation fare/ avanzare/formulare una proposta, einen Vorschlag machen/unterbreiten/formulieren'. Die Basis wählt einen der möglichen Kollokationspartner aus, um den notwendigen Inhalt auszudrücken. Laut Konecny ist die Bestimmung des unilateralen und bilateralen Verhältnisses nicht immer einfach. Es gibt keine klare Grenze und daher handelt es sich um ein Kontinuum, auf dem sich Kollokationen befinden. Ob unilaterales oder bilaterales Kollokationsverhältnis, Basis und Kollokator bedingen sich immer gegenseitig.

Eng mit der Implikation und Ausgerichtetheit ist auch die Assoziativität verbunden. Im Bereich der Lexikologie handelt es sich um das unbewusste Verknüpfen sprachlicher Zeichen auf der Grundlage psychologischer Prozesse (Donalies 1994: 342). Die Assoziation von lexikalischen Einheiten im mentalen Lexikon und die Verbindung der gespeicherten Konzepte führen nämlich zu assoziativen Verbindungen. Die Assoziativität der Kollokationen ist nicht einseitig ausgerichtet, sie ist reversibel. Das Lexem melken assoziiert das Wort Kuh, aber das Lexem Kuh muss nicht unbedingt melken assoziieren (Gladysz 2003: 62). So ist zum Beispiel das Adjektiv blond direkt mit Haar verbunden, also handelt es sich um ein Lexem mit hohem Grad an Assoziativität. Auf der anderen Seite hat das Lexem Haar ein größeres syntagmatisches Potenzial und der Grad der Assoziativität ist demgemäß geringer. Somit ist der Grad der Assoziativität 
indirekt proportional zum Umfang des Kollokationspotenzials. Die Assoziativität selbst kann durch die außersprachliche Realität bedingt sein, in der bestimmte Objekte, Eigenschaften oder Tatsachen miteinander verbunden sind oder durch innersprachliche Konvention, die vorgibt, dass bestimmte Lexeme mit anderen gemeinsam vorkommen.

\section{SCHLUSSFOLGERUNG}

Die Betrachtung der Kollokation als semantisch asymmetrische Verbindung verdeutlicht, dass diese spezifische Art von Mehrwortverbindung durch eine Reihe unterschiedlicher Eigenschaften gekennzeichnet ist, die sich gegenseitig bedingen und im unterschiedlichen Maße in einer Kollokation ausgeprägt sind. Die Bedeutung der Basis kann unabhängig vom Kollokator bestimmt werden, was sie aus semantischer Sicht relativ unveränderlich macht. Diese Stabilität im semantischen Sinne ermöglicht die relativ transparente Bedeutung der Kollokation. Somit können Kollokationsverbindungen als kompositionell oder semikompositionell charakterisiert werden. Der Kollokator hingegen stellt eine in semantischer Hinsicht variable Komponente der Kollokationsverbindung dar. Er weist einen bestimmten Grad an Polysemie auf, weil er in verschiedenen Kollokationen unterschiedliche Bedeutungen haben kann, die erst in der Verbindung mit der Basis disambiguiert werden können. Die Verbindung mit der Basis selbst hängt von semantischen Einschränkungen, den sog. Kollokationsrestriktionen, ab. Sie können nicht nur durch die Kompatibilität der semantischen Merkmale erklärt werden, da sich Kollokationsmuster nicht wie semantische Klassen verhalten. Handelt es sich um Verbindungen von Lexemen, die nicht mindestens in einem semantischen Merkmal miteinander übereinstimmen, kann die Untersuchung aus diachroner Perspektive dazu beitragen, die Natur der Verbindung zu bestimmen. Die Bedeutung des Kollokators kann mit der Zeit Bedeutungsveränderungen durchlaufen, sodass die Bedeutung kollokationsintern nicht (mehr) mit der kollokationsexternen Bedeutung übereinstimmt. Vom Grad der semantischen Modifikation des Kollokators hängt auch die semantische Motivation ab. Je höher der Grad der semantischen Modifikation des Kollokators, desto geringer die Motivation und desto höher die Idiosynkrasie.

Aus synchroner Perspektive hängt die Bedeutung des Kollokators von der Stärke des semantischen Zusammenhalts zwischen Kollokator und Basis ab. Je enger die Bedeutung, desto stärker die semantische Kohäsion und desto geringer das Kollokationspotenzial. Die semantische Kohäsion beeinflusst auch die Kollokationsrestriktionen. Die übertragene Bedeutung, die sich aus der Kollokationsverbindung ableitet, führt zu Restriktionen im Bereich der paradigmatischen Substituierbarkeit. Die Kohäsionsstärke hängt somit von der Bedeutungsveränderung ab. Je höher die Kohäsion, desto weiter hat sich die kollokationsinterne Bedeutung von der der kollokationsexternen Bedeutung entfernt. Es liegt somit der Schluss nahe, dass der Grad der Festigkeit einer Mehrwortverbindung in Abhängigkeit zur Kohäsionsstärke bestimmt werden kann. Dies weist auf den äußerst graduellen Charakter der Kollokationsverbindung hin, die sich deshalb je nach Kohäsionsgrad auf einem Kontinuum zwischen freien Wortverbindungen und Phraseologismen befindet. Je stärker die Kohäsion, desto näher liegt die Kollokation an den Phraseologismen. 
Abschließend lässt sich festhalten, dass Kollokationen als dynamisches Phänomen aufzufassen sind und dass gerade diese Tatsache ihre eindeutige Bestimmung geradezu unmöglich macht.

\section{Literatur}

BAHNS, Jens (1996) Kollokationen als lexikographisches Problem. Eine Analyse allgemeiner und spezieller Lernerwörterbücher des Englischen. Tübingen: Niemeyer.

BARTSCH, Sabine (2004) Structural and Functional Properties of Collocations in English. A Corpus Study of Lexical and Pragmatic Constraints on Lexical Co-Occurrence. Tübingen: Narr.

BORIĆ, Neda (2000) „Kompleksni jezični znakovi s osvrtom na praktične probleme njihova prevođenja." Strani jezici 29/4, 201-208.

DONALIES, Elke (1994) „Idiom, Phraseologismus oder Phrasem? Zum Oberbegriff eines Bereichs der Linguistik." Zeitschrift für germanistische Linguistik 22, 334-349.

DUDEN-ONLINEWÖRTERBUCH www.duden.de [12.11.2018].

GLADYSZ, Marek (2003) Lexikalische Kollokationen in deutsch-polnischer Konfrontation. Frankfurt a. Main: Peter Lang Verlag.

HAUSMANN, Franz-Josef (1984) „Wortschatzlernen ist Kollokationslernen. Zum Lehren und Lernen französischer Wortverbindungen." Praxis des neusprachlichen Unterrichts 31, 395-406.

HAUSMANN, Franz-Josef (1985) „Kollokationen im deutschen Wörterbuch. Ein Beitrag zur Theorie des lexikographischen Beispiels.“ In: H. Bergenholtz/J. Mugdan (Hrsg.), Lexikographie und Grammatik. Akten des Essener Kolloquiums zur Grammatik im Wörterbuch 28. - 30.6.1984. Tübingen: Niemeyer Verlag, 118-129.

HAUSMANN, Franz-Josef (1993) „Ist der deutsche Wortschatz lernbar? Oder: Wortschatz ist Chaos." Info DaF 20/5, 471-485.

HAUSMANN, Franz-Josef (2004) „Was sind eigentlich Kollokationen?“ In: K. Steyer (Hrsg.), Wortverbindungen mehr oder weniger fest. Jahrbuch des Instituts für Deutsche Sprache Mannheim 2003. Berlin: de Gruyter, 309-334.

HRVATSKI JEZIČNI PORTAL (Kroatisches Sprachportal/Online-Wörterbuch) http:// hjp.znanje.hr/ [12.11.2018].

ILIESCU, Maria (2006) „Kollokationen in den romanischen Sprachen.“ In: W. Dietrich et al. (Hrsg.), Lexikalische Semantik und Korpuslinguistik. Tübingen: Narr, 189-208.

KOHN, Kurt (1992) „Bemerkungen zur Kollokationsproblematik.“ In: H. Klaus/S. R. Anschütz (Hrsg.), Texte, Sätze, Wörter und Moneme. Festschrift für Klaus Heger zum 65. Geburtstag. Heidelberg: Heidelberger Orientverlag, 369-387.

KONECNY, Christine (2003) Divergenze e convergenze in collocazioni italiane e tedesche. Magisterarbeit. Innsbruck: Universität Innsbruck.

KONECNY, Christine (2010) Kollokationen: Versuch einer semantisch-begrifflichen Annäherung und Klassifizierung anhand italienischer Beispiele. München: Martin Meidenbauer.

LEHR, Andrea (1996) Kollokationen und maschinenlesbare Korpora. Ein operationales Analysemodell zum Aufbau lexikalischer Netze. Tübingen: Niemeyer 
PALMER, Frank (1976) Semantics. Cambridge: Cambridge University Press.

PRITCHARD, Boris (1998) „O kolokacijskom potencijalu rječničkog korpusa.“ Filologija 30-31, 285-304.

REDER, Anna (2006) Kollokationen in der Wortschatzarbeit. Wien: Praesens Verlag. STOJIĆ, Aneta/Sanela MURICA (2010) „Kolokacije - teorijska razmatranja i primjena u praksi na primjerima iz hrvatskoga i njemačkoga jezika." Fluminensia 22/2, 111-126. STOJIĆ, Aneta (2012) Kolokacije - prilog teoriji i praksi. Rijeka: Sveučilište u Rijeci. STOJIĆ, Aneta/Nataša KOŠUTA (2012) „Zur Abgrenzung von Mehrwortverbindungen.“ Zagreber germanistische Beiträge 21/2, 359-373.

STOJIĆ, Aneta/Tamara ŠTIGLIĆ (2012) „Kollokationen im deutsch-kroatischen Sprachvergleich.“ Jezikoslovlje 12/2, 263-282.

STOJIĆ, Aneta (2014) „Kollokationen in der Sprach- und Kulturmittlung (am Beispiel des Sprachenpaares Deutsch-Kroatisch). In: A. Đurović/V. Kučiš (Hrsg.), Translation und transkulturelle Kommunikation. Beograd: Philologische Fakultät der Universität Belgrad: 81-95.

TEUBERT, Wolfgang (1999) „Korpuslinguistik und Lexikographie.“ Deutsche Sprache 4, 293-313.

\section{Zusammenfassung \\ ZUR SEMANTIK VON KOLLOKATIONEN}

Als eine spezifische Art von Wortverbindungen stellen Kollokationen trotz zahlreicher einschlägiger Untersuchungen noch immer eine Herausforderung aus deskriptiver Perspektive dar. Das betrifft insbesondere die lexikalisch-semantische Ebene. Diese syntagmatischen Wortkombinationen, die zusammen eine Einheit bilden, unterscheiden sich wegen ihrer Unauffälligkeit und semantischen Transparenz auf den ersten Blick nicht von anderen sprachlichen Sequenzen. Vergleicht man sie aber mit gleichwertigen Verbindungen aus anderen Sprachen, erscheinen oftmals gewisse Unterschiede. Die Art und Weise, wie man Kollokationen bildet, und somit auch die Möglichkeit der Bildung neuer Bedeutungen, die aus einer bestimmten Wortkombination ergeht, ist nämlich jeder Sprache immanent. Kollokationen sind folglich einzelsprachspezifisch. Mithilfe einer funktionalen strukturell-syntaktischen Sicht auf die Kollokationsbestandteile sollen im Beitrag die semantische Motivation, die semantische Kohäsion zwischen den Bestandteilen der Kollokation sowie die semantischen Modifikationen des Kollokators beleuchtet werden. Da die Spezifik der inhaltlichen Beziehungen oftmals erst im Sprachvergleich auf der lexikalischen Ebene deutlich wird, werden einzelne semantische Charakteristika am Beispiel von Kollokationen unterschiedlicher Sprachen illustriert. Ziel des Beitrages ist es, die ambivalente inhaltliche Seite der Kollokationen aufzudecken, um somit zu einem besseren Verständnis dieser sprachlichen Erscheinung beizutragen.

Schlüsselwörter: Kollokationen, Basis, Kollokator, Idiosynkrasie, semantische Kohäsion, semantische Verträglichkeit, syntagmatische Beschränkung 


\section{Abstract \\ A CONTRIBUTION TO THE SEMANTICS OF COLLOCATIONS}

As a specific type of multi-word expressions, collocations still pose a major challenge from a descriptive perspective, despite numerous existing studies. This is especially noticeable at the lexico-semantic level. These syntagmatic word combinations, which form a unity, differ from other linguistic sequences because of their apparent semantic inconspicuousness. However, by comparing them with equivalent multi-word expressions in other languages, some important differences become evident. This relates in particular to the formation of collocations, and thus to the creation of new meanings emanating from particular word combinations, which are language specific. Adopting a functional structural-syntactic view of the collocation components, our goal is to investigate the semantic motivation as well as the semantic cohesion between the elements of the collocation and the possible semantic modifications involved. Since the specificity of the semantic relations often becomes lexically evident only at a cross-linguistic level, examples of collocations in different languages are provided. The aim of the paper is to present the ambivalent character of the collocations and contribute to a clearer understanding of the linguistic phenomenon in question.

Key words: collocations, base; collocate, idiosyncracy, semantic cohesion, semantic compatibility, syntagmatic restriction

\section{Povzetek \\ K SEMANTIKI KOLOKACIJ}

Kljub številnim raziskavam so kolokacije kot specifična vrsta besednih zvez z deskriptivnega vidika še vedno izziv, in sicer predvsem na leksikalno-semantični ravnini. Te sintagmatične besedne kombinacije, ki skupaj tvorijo enoto, se zaradi neopaznosti in pomenske transparentnosti na prvi pogled ne razlikujejo od drugih jezikovnih sekvenc. Če pa jih primerjamo z enakovrednimi zvezami iz drugih jezikov, je pogosto opaziti določene razlike. Način, kako se tvorijo kolokacije, s tem pa tudi možnost nastajanja novih pomenov, ki se razvije iz določenih besednih kombinacij, je namreč imanentna vsakemu jeziku posebej. Zato so kolokacije jezikovno specifične. V prispevku je s pomočjo funkcionalno strukturalno-skladenjskega pristopa osvetljena pomenska motivacija kolokacijskih sestavin, pomenska kohezija med njimi ter pomenske modifikacije kolokatorja. Ker se specifika vsebinskih razmerij pogosto osvetli šele z jezikovno primerjavo na leksikalni ravnini, so posamezne pomenske lastnosti prikazane s primerom kolokacij iz različnih jezikov. Cilj prispevka je prikazati ambivalentno vsebinsko plat kolokacij, da bi tako prispevali k boljšemu razumevanju teh jezikovnih pojavov.

Ključne besede: kolokacije, osnova, kolokator, idiosinkrazija, pomenska kohezija, pomenska kompatibilnost, pomenske restrikcije 